\title{
Unsupervised Prediction of Channel State for Cognitive Radio Using Hidden Markov Model
}

\author{
Honghao Wei, Yunfeng Jia, Lin Qiu \\ School of Electronic and Information Engineering, \\ Beihang University, Beijing, China \\ whhbuaa@foxmail.com
}

\author{
Yishuai Zhu \\ Beijing Military Represent Bureau, Army Aviation PLA, \\ Beijing, China \\ plazys@sina.com
}

\begin{abstract}
The accurate modeling of primary users (Pus) behavior is important and crucial to cognitive radio (CR). The method to detect idle frequencies, not used by primary users' (Pus') has been widely investigated recent years. Existing researches need to estimate and select the threshold of the energy detector manually. In this paper, we propose an unsupervised approach to estimate channel states. We adopt different number of observed state according to different classification in hidden Markov model (HMM). We trained and tested the model through experiments using real spectrum measurement data. The system we proposed can automatically deal with large amounts of data and present high performance and good expansibility to predict channel state.
\end{abstract}

Keywords-Component; Hidden Markov model; Cognitive Radio; Unsupervised Prediction

\section{INTRODUCTION}

Spectrum resource is non-renewable. To better utilize the spectrum source, Cognitive radio technology emerges, and has been extensive research to find a method to share the licensed spectrum. The cognitive radio senses a given channel for idle time of the primary or licensed users, to prevent the harmful interference when a secondary user try to access the channel during such intervals [1]. In general spectrum sensing, the focus is on modeling of spectrum occupancy and predicting the status of Pus as being active or idle using different kinds of sensing techniques[2][3].

Recently, many researches have focused on HMM approach to predict Pus channel state. For examples, a realtime measurements collected in the paging band were used to validate the on-off behavior of Pus has been introduced in [4] In [5], a spectrum occupancies predicting approach based on a non-stationary hidden Markov model was proposed. Nguyen et al. proposed a hidden bivariate Markov model to characterize the transmission behavior of a PU [6]. However, most of the previous works identified the idle and busy states of a channel by selecting a threshold for the energy detector from a histogram of the power levels (in $\mathrm{dBm}$ ). And the threshold was set based on the noise level of the spectrum band and noise figure of the receiver. This paper puts forward an unsupervised prediction of channel state based on the k-means algorithm and multi-peaks Gaussian fitting without selecting a threshold manually. Besides some of the previous works only concerned about two observation states in a basic or an expansion HMM, while others defined the observation through an normally distribution [7]. However,

This paper is sponsored by National Natural Science Foundation of China NO.61371007. the histograms of the power in most channels are various from each other and not only have two independent Gaussian signal areas. So a single threshold is no longer applicable, and the prediction effect of the channel states is unsatisfactory with the number of the single in the histogram of the power increasing. Our approach identify different number of observation states through different classification by selecting multiple thresholds. Relying on real spectrum measurement data, the parameters of model is estimated by Baum-Welch algorithm. Given these parameters, the state of activity of the Pus at any time and frequency band is predicted using the forward-backward algorithm. We have carefully implemented and tested our model rely on real spectrum measurement data. Our system show a good performance in different situations.

The remainder of the paper is organized as follows. An unsupervised prediction of channel state model for spectrum sensing is given is Section II, Section III discusses the system performance evaluation. Concluding remarks are given in Section IV.

\section{SYSTEM MODEL}

The configuration of the proposed unsupervised prediction of channel state system for dynamic spectrum access is shown in Figure1. The system consists of three main components: channel analyzing and classifying, channel parameter estimator, channel state estimator and predictor. The channel analyzing and classifying selects the channels which are not busy or idle all the time as our objects of study and classifies them into different types. The channel parameter estimator estimates the HMM parameter for different types. The channel state estimator and predictor uses the estimated HMM parameter to estimate the hidden state as a base of channel access policy.

\section{A. Channel Analyzing and Classifying}

In this system, the data of spectrum channels are classified and fitted according to the k-means algorithm and multi-peaks Gaussian fitting. We first define a maximum classification $\mathrm{M}$ and decrease it step by step to get the final classification $\mathrm{N}$ according to two criterions, the first is that the absolute difference of two classifications' centers should be less than the threshold $r_{c}$, ant the other is that the number of data in a classification should be more than the threshold $r_{n}$. Set the two criterions is to combine two similar kinds and cut down the unnecessary classification. The center of 
each classification is set as the initial mean value of the multi-peaks Gaussian fitting method to fit the spectrum measurements. The multi-peaks Gaussian fitting function is described as

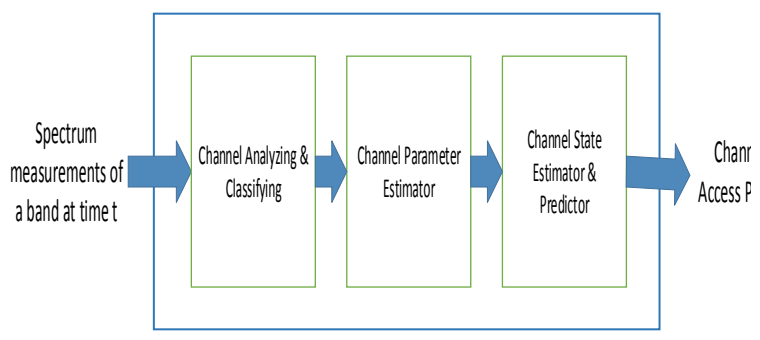

Figure 1. System model for unsupervised prediction of channel state in CR

$$
f(x)=\sum_{i=1}^{n} c_{i} * \exp \left(\frac{-\left(x-\mu_{i}\right)^{2}}{2 \sigma_{i}^{2}}\right)
$$

\section{B. Maintaining Channel Parameter Estimator}

In this paper, we denote a discrete-time Markov chain by $\left\{Y_{t}, S_{t}, t=0,1, \ldots\right\}$. The process $\mathrm{St}$ is the status of a licensed channel by a set $S=\{1,2\}$ with 1 representing the idle state of the channel and 2 its busy state, while $\left\{Y_{t}\right\}$ is referred to as the observable process and its value is obtained from real spectrum measurements. The hidden process $\{\mathrm{St}$, is a discrete-time finite state homogeneous Markov chain. The random observable $\left\{Y_{t}\right\}$ are conditionally independent given $\left\{S_{t}\right\}$. Using the curve fitting method, we get two groups of data, one on mean $\mu_{i}$ and the other on the variance $\sigma_{i}$ from function (1). We sort the results computed by (2) from lowest to highest to get the multi-thresholds. A given channel with $\mathrm{n}$ classification has $2 * \mathrm{~N}$ thresholds.

$$
\begin{aligned}
& t h_{i}=\mu_{i}+3 * \sigma_{i}(i=1,2 \ldots, N) \\
& t h_{i}^{\prime}=\mu_{i}-3 * \sigma_{i}(i=1,2 \ldots, N)
\end{aligned}
$$

\footnotetext{
$A=\left\{a_{i j}, i, j \in S\right\}$ denote the transition matrix of $\left\{S_{t}\right\}$,
}

$$
\begin{aligned}
& (\mathrm{A})_{\mathrm{i}, \mathrm{j}}=b_{i j}=\operatorname{Pr}\left(X_{t+1}=j \mid \mathrm{X}_{t}=i\right) \\
& i, j=1,2
\end{aligned}
$$

Let $\pi_{i}=P\left(S_{1}=i\right), i \in S$, denote the probability that initial state is $i$. We define the observed state sequence $\left\{O_{t}, t=0,1, \ldots\right\}$ by:

$$
O_{t}=\left\{\begin{array}{l}
1 \quad Y_{t}<t h_{1} \\
2 \quad t h_{2}^{\prime}<Y_{t}<t h_{2} \\
\cdots \cdots \\
n \quad t h_{n}^{\prime}<Y_{t}<t h_{n} \\
N+1 \quad \text { else }
\end{array}\right.
$$

Let $\quad$ set $\quad \mathrm{O}=\{1,2, \ldots N+1\}$
And $B_{j k}=B=\left\{b_{j k}, j \in S, k \in O\right\} \quad$ is the emission probability matrix,

$$
\begin{aligned}
& (\mathrm{B})_{j, k}=b_{j k}=\operatorname{Pr}\left(O_{t}=k \mid \mathrm{X}_{t}=j\right) \\
& j=1,2 \\
& k=1, \ldots, N+1
\end{aligned}
$$

The HMM for a given channel is the characterized by the parameter $\lambda=(\pi, A, \mathrm{~B})$.

Now we proceed to estimate the parameters of a HMM based on a sequence of hidden states. $s^{T}=\left\{s_{t}, t=1, \ldots, T, s_{t} \in S\right\}$ and a sequence of observations, $o^{T}=\left\{o_{t}, t=1, \ldots, T, o_{t} \in O\right\}$.

The parameter estimation can be done by Baum-Welch algorithm [9]. For a given $\lambda$, the parameters are reestimated and the HMM update with a new $\hat{\lambda}$.

We define $q_{t}(j)=P\left(s_{t}=j \mid o^{T}, \lambda\right)$ to be the probability of being in state ${ }^{S_{i}}$ at time t given the observation $o_{t}, \quad q_{t}(i, j)=P\left(s_{t}=i, s_{t+1}=j \mid o^{T}, \lambda\right)$ to be the probability of being in state $S_{i}$ at time t and transiting to state ${ }^{S_{j}}$ at time $\mathrm{t}+1$ given ${ }^{o_{t}}$ under the parameter $\lambda$. The expected number of transition from state ${ }^{S_{i}}$ during the path is given by 


$$
\begin{aligned}
& E\left(q_{t}(i)\right)=\sum_{t=1}^{T-1} q_{t}(i) \\
& E\left(q_{t}(i, j)\right)=\sum_{j=1}^{T-1} q_{t}(i, j)
\end{aligned}
$$

The model parameters of HMM from a training sequences can be estimated as $\hat{\lambda}$. The estimation formulas are given by

$$
\begin{gathered}
\hat{\pi}_{i}=q_{1}(i) \\
\hat{a}_{i j}=\frac{E\left(q_{t}(i, j)\right)}{E\left(q_{t}(i)\right)}=\frac{\sum_{j=1}^{T-1} q_{t}(i, j)}{\sum_{j=1}^{T-1} q_{t}(i)} i, j=1,2 \\
\hat{b}_{j k}=\frac{\sum_{o_{t}=k}^{T} q_{t}(j)}{\sum_{t=1}^{T} q_{t}(j)} j=1,2, k=1,2, \ldots, N+1
\end{gathered}
$$

Then we get the new parameter of HMM described as $\hat{\lambda}=(\hat{\pi}, \hat{A}, \hat{B})$, this algorithm generates a sequence of HMM parameter estimates with non-decreasing likelihood values. Every time we iterate the Baum algorithm starts with a current parameter $\lambda_{l}$ and estimate a new parameter $\lambda_{l+1}$. The algorithm is terminated when a convergence criterion is satisfied.

\section{Channel State Estimator and Predictor}

Spectrum sensing detects the availability of the spectrum, which is essential and important to CR. The goal of the spectrum sensing is to accurately predict the state of a given channel with low probability of false alarm and high probability of detection. Predicting the channel state at one time step ahead the current state based on a sequence of hidden states, $s^{T}=\left\{s_{t}, t=1, \ldots, T, s_{t} \in S\right\}$ and a sequence of observations, $o^{T}=\left\{o_{t}, t=1, \ldots, T, o_{t} \in O\right\}$ can be solved using hidden Markov model Viterbi algorithm [8].

We let $\delta_{t}(i)$ to be the maximal probability of state sequences of the length $\mathrm{T}$ that end in state ${ }^{i}, \delta_{t}(i)$ can be written as

$$
\delta_{t}(i)=\max _{S_{1}, \ldots, \mathrm{s}_{t-1}}\left\{P\left(s_{1}, \ldots, \mathrm{s}_{t}=i ; \mathrm{o}_{1}, \ldots, \mathrm{o}_{t} \mid \lambda\right)\right\}
$$

Let $\phi_{t}(i)$ to be the array that store the parameters that maximize $\delta_{t}(i)$. The initial value of the intermediate variable is

$$
\begin{aligned}
& \delta_{1}(i)=\pi_{i} b_{i}\left(o_{1}\right), \quad 1 \leq i \leq 2 \\
& \phi_{1}(i)=0, \quad 1 \leq i \leq 2
\end{aligned}
$$

The recursive computation of $\delta_{t}(j)$ and $\phi_{t}(j)$ can be done by

$$
\begin{aligned}
& \delta_{t}(j)=\max _{1 \leq j \leq n}\left[\delta_{t-1}(i) a_{i j}\right] b_{j}\left(o_{t}\right), 2 \leq t \leq T \\
& 1 \leq j \leq 2 \\
& \phi_{t}(j)=\underset{1 \leq i \leq n}{\arg \max }\left[\delta_{t-1}(i) a_{i j}\right], 2 \leq t \leq T \\
& 1 \leq j \leq 2
\end{aligned}
$$

Then we can get the calculated likelihood probability $P^{*}$ and the estimated state at $\mathrm{T} q_{T}^{*}$.

$$
\begin{array}{r}
P^{*}=\max _{i=1,2}\left[\delta_{T}(i)\right] \\
q_{T}^{*}=\underset{i=1,2}{\operatorname{argmax}}\left[\delta_{T}(i)\right]
\end{array}
$$

The estimating of the hidden state in a given channel is

$$
q_{t}^{*}=\phi_{t+1}\left(q_{t+1}^{*}\right), \quad t=T-1, T-2, \ldots, 1
$$

The channel access policy makes decision based on the outcome from the channel state estimator and predictor.

\section{PERFORMANCE ANALYSIS}

In this section, we carry out some numerical experiments based on real spectrum measurement data to evaluate the performance of the CR system discussed in Section II .

\section{A. Channel Parameter Estimation with Real Spectrum Measurements}

The data used in this paper were collected through a spectrum analyzer on the rooftop of the New Main Building at Beihang University, from 00:00:00 March 18, 2015 to 00:00:00 March 22, 2015. We randomly study the 88-128 $\mathrm{MHz}$ band with a frequency resolution of $100 \mathrm{KHz}$ and a time resolution of 10 seconds. The system model in the proposed method based on day spectrum measurements at random from the five days' data. Figure 2 shows the occupancy spectrum measurements over 24 hours in March 
19 in the selected band. From the figure, we can see that most of the channels are being utilized but not all channels are occupied all the time. The number of observation symbols for this channel is 8640 .

We analyzed and classified the measurements into three classifications depend on the value $\mathrm{n}$ obtained by the method in Section II based k-means algorithm in each channel. The thresholds $r_{c}$ and $r_{n}$ influence the precision of the number of the classifications. In this paper we defined $r_{c}=1$ and $r_{n}=800$. Figure 3 shows histogram of the different classifications. Figure4 shows the histogram plots of the channels we randomly selected in four representative types. The left figure of classification 1 demonstrates the channel is always utilized and the right one shows a channel barely occupied. The method proposed in this paper focus on estimating the states of the channels which both have noise and signal as classification 2 and classification 3 in the histogram plots shows.

In this section, we only focus on HMM parameter estimation for a single channel. The parameters of other channels in the band were estimated similarly. We choose the channel from classification 3 as is shown in Figure 3 to be analyzed. Given the measurements of the channel, we fitted the histogram of power by (1) with the initial value (51.7892, -57.2836, -75.6072) computed by the k-means algorithm. Figure5 shows the fitting result of the histogram of power.

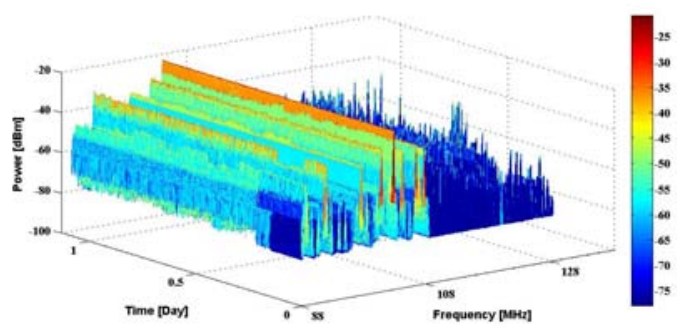

Figure 2. Three-dimensional view of received powers

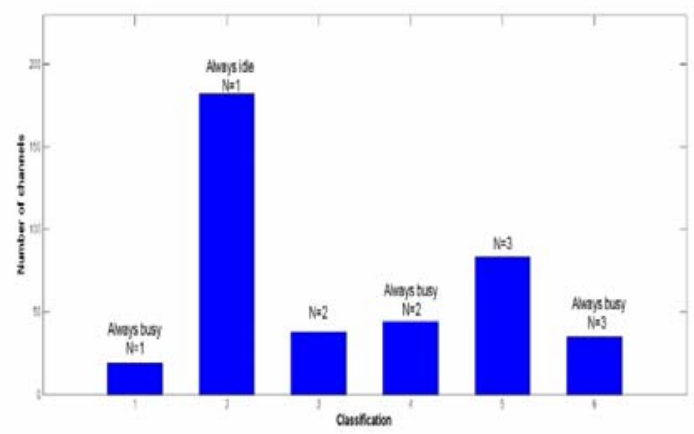

Figure 3. The number of different classifications
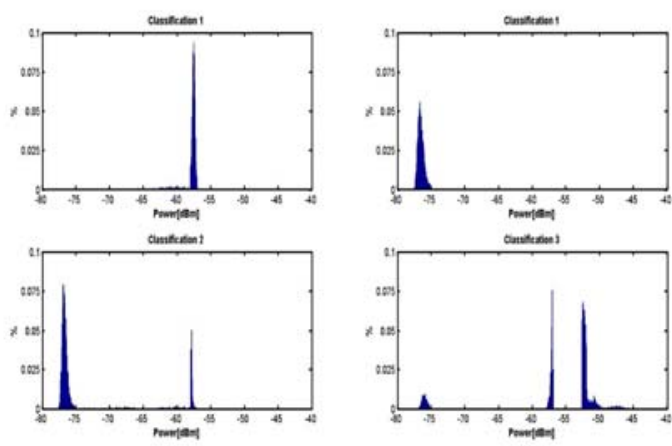

Figure 4. Histogram Plots from different types

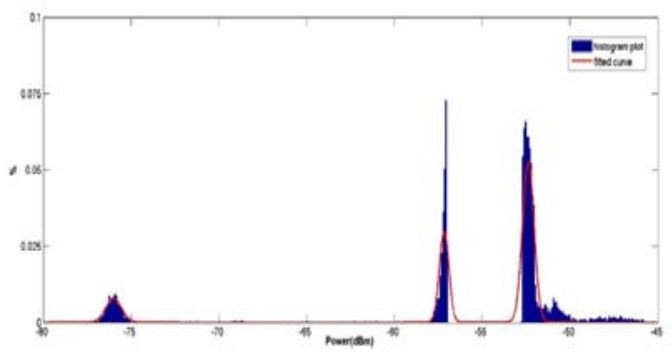

Figure 5. Fitting result

The parameters of the multi-peaks Gaussian fitting function is TABLE I

The thresholds of the given channel is in (19). Then we can get the observable sequences based on the thresholds. Relying on the measurements of the channel, we have used the algorithm described in Section II and estimates the HMM parameters. The proposed algorithm was terminated if the relative difference in log-likelihood values of the last two iterations was smaller than10-4.

TABLE I. PARAMETERS OF THE MULTI-PEAKS GAUSSIAN FITTING FUNCTION

\begin{tabular}{|c|c|c|}
\hline$c_{1}=0.09261$ & $\mu_{1}=-75.99$ & $\sigma_{1}=0.4464$ \\
\hline$c_{2}=0.3559$ & $\mu_{2}=-57.17$ & $\sigma_{2}=0.2661$ \\
\hline$c_{3}=0.6306$ & $\mu_{3}=-52.38$ & $\sigma_{3}=0.3249$ \\
\hline
\end{tabular}

$t h_{1}=-74.6492, t h_{2}=-56.3721, t h_{3}=-51.4016$

$t h_{1}^{\prime}=-77.3274, t h_{2}^{\prime}=-57.9685, t h_{3}^{\prime}=-53.3511$

The estimated HMM parameter set is shown in (20), (21) and (22), which is consistent with measurement plots.

$$
\hat{\pi}=[1,0]
$$

$$
\hat{A}=\left[\begin{array}{cc}
0.99 & 0.01 \\
0.0011 & 0.9989
\end{array}\right]
$$




$$
\hat{B}=\left[\begin{array}{cccc}
0.9849 & 1.1524 e-08 & 1.9306 e-13 & 0.0151 \\
0 & 0.2954 & 0.5699 & 0.1347
\end{array}\right]
$$

\section{B. Channel State Estimation and Prediction}

We use the real spectrum measurement of the same given channel in another day to test our system. Since the state occupancy sequence for the real data is not known, it is necessary to apply our system to estimate the hidden state sequence as the real data first. The steps were carried out as follow:

1) Generate a hidden state sequence $\tilde{S}^{T}=\left\{\tilde{s}_{t}, t=1, \ldots, T, \tilde{s}_{t} \in S\right\}$ using the estimated initial distribution $\hat{\pi}$ and the transition matrix $\hat{A}$.

2) Randomly select the same channel spectrum measurement of another day, and generate the observation sequence $\tilde{o}^{T}=\left\{\tilde{o}_{t}, t=1, \ldots, T, \tilde{o}_{t} \in O\right\}$ with the method proposed in Section II.

3) Re-estimate the parameter set $\tilde{\lambda}$ of the channel from the observation sequence in Step 2). We obtained the parameter set $\tilde{\lambda}$ :

$$
\begin{gathered}
\tilde{\pi}=[1,0] \\
\tilde{A}=\left[\begin{array}{lll}
0.9912 & 0.0088 \\
0.0009 & 0.9991
\end{array}\right] \\
\tilde{B}=\left[\begin{array}{cccc}
0.9753 & 4.4904 e-18 & 0 & 0.0247 \\
0 & 0.2699 & 0 & 0.7301
\end{array}\right]
\end{gathered}
$$

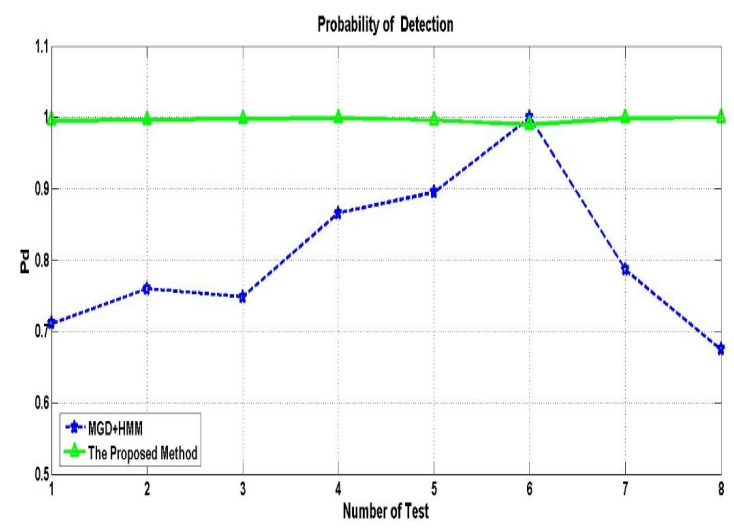

Figure 6. Comparison of Probability of Detection

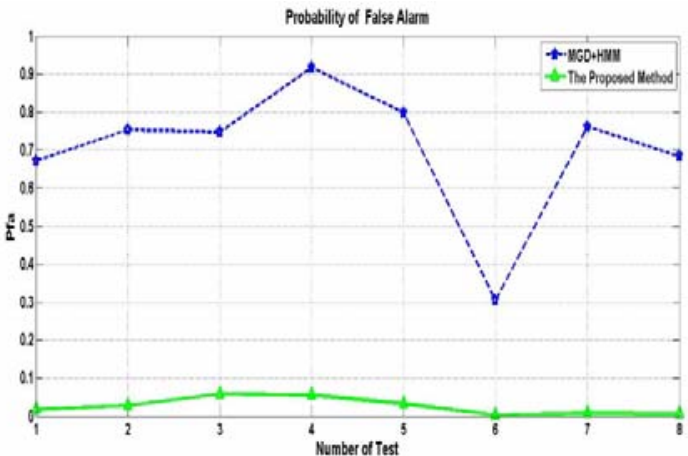

Figure 7. Comparison of Probability of False Alarm

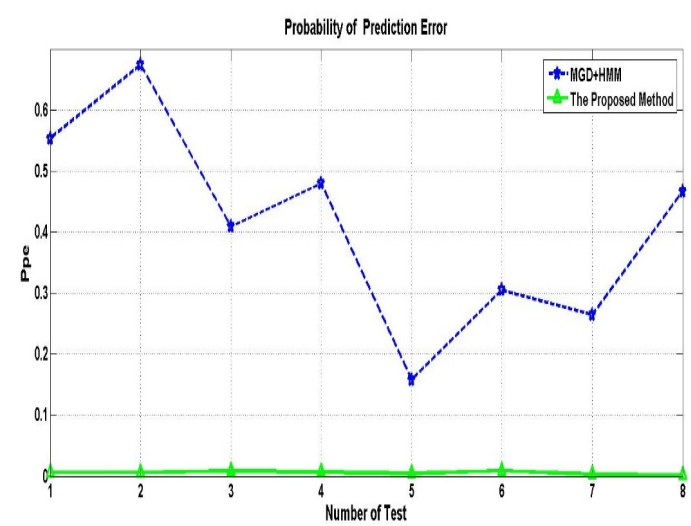

Figure 8. Comparison of Prediction Error

4) Use the new estimated parameter set $\tilde{\lambda}$ and the observation sequence $\tilde{O}^{T}$ to predict the state.

5) Let $P_{d}$ to be the probability of detection, $P_{f a}$ to be the probability of false alarm and $P_{p e}$ to be the probability of prediction error. The probability of false alarm indicates that when the idle channel is detected as busy.

The values of $P_{d}, P_{f a}$ and $P_{p e}$ is

$$
P_{d}=0.9961, P_{f a}=0.0112, P_{p e}=0.0046
$$

We randomly selected eight channels in the classification with $\mathrm{N}=3$ which are not always used. Comparing the approach proposed in this paper and a combination method of HMM and multivariate Gaussian distribution (MGD) in [7] to estimate the channel state, Figure6 , Figure7 and Figure8 show the comparison of $P_{d}, P_{f a}$ and $P_{p e}$. The system proposed in this paper have very good performance.

\section{CONCLUSION}

In this paper, we present an unsupervised prediction of channel state. Since the true channel states cannot be exactly known and the only information we may obtain is the observations of received signal strength. The method we proposed to classify the channels is more timesaving and 
intelligent than ever before. Base on the classification and initial value, the model can be used for channel occupancy prediction. The experiments show good flexibility of the proposed system and evaluate the prediction performance.

\section{REFERENCES}

[1] J. Unnikrishnan and V. Veeravalli, "Cooperative sensing for primary detection in cognitive radio," IEE J. Sel. Topics Signal Process. , vol. 2, no. 1, pp. 18-27, Feb. 2008.

[2] D. Chen, S. Yin, Q. Zhang, M. Liu, and S. Li, "Mining spectrum usage data: A large-scale spectrum measurement study," in Proc. ACM MobiCom, Beijing, China, Sep. 2009, pp. 13-24.

[3] J. J. Lehtomäki, R. Vuohtoniemi, and K. Umebayashi, "On the measurement of duty cycle and channel occupancy rate,” IEEE J. Sel. Areas Commun., vol. 31, no. 11, pp. 2555-2565, Nov. 2013.

[4] C. Ghosh and D. P. Agrawal, "Markov chain existence and hidden Markov models in spectrum sensing,” Proc. IEEE PerCom’09, pp. 26, 2009.
[5] Xianfu Chen, Honggang Zhang, Allen B. MacKenzie, and Marja Matinmikko, "Predicting Spectrum Occupancies Using a NonStationary Hidden Markov Model,” IEEE Wireless Commu., vol. 3, issue. 4, pp. 333-336, Aug. 2014.

[6] T. Nguyen, B. L. Mark, and Y. Ephraim, "Spectrum sensing using a hidden bivariate Markov model," IEEE Trans. Wireless Commun., vol. 12, no. 9, pp. 4582-4591, Sep. 2013.

[7] T. Nguyen, B. L. Mark, and Y. Ephraim, "Hidden Markov process based dynamic spectrum access for cognitive radio," in Proc. Conf. Inf. Sci. Syst. (CISS), Baltimore, MD, USA, Mar. 2011.

[8] L. R. Rabiner, "A Tutorial on Hidden Markov Models and Selected Applications in Speech Recognition." Proc. Of the IEEE, Vol.77, No.2,pp.257-286,1989.

[9] Eelch, Lloyd R. "Hidden Markov models and the Baum-Welch algorithm." IEEE information Theory Society Newsletter 53.4 (2003):10-13. 